\title{
Cytomegalovirus retinitis after the initiation of highly active antiretroviral therapy: a 2 year prospective study
}

Suzanne M Mitchell, William L Membrey, Michael S Youle, Adanna Obi, Simon Worrell, Brian G Gazzard

\begin{abstract}
Backgroundlaims-There have been several recent reports suggesting that the natural history of cytomegalovirus retinitis (CMVR) has been significantly modified with the development of highly active antiretroviral therapy (HAART). This 2 year prospective cohort study assesses the effect of HAART on the incidence and progression of $\mathrm{CMV}$ retinitis in patients with CD4 cell counts below 50 cells $\times 10^{6} / 1$. Methods-63 patients, with CD4 cell counts below 50 cells $\times 10^{6} / 1$, who were recruited to a 2 year prospective cohort study at the commencement of combination antiretroviral therapy including the use of the proteinase inhibitor, indinavir, were reported. The response to HAART was assessed in terms of a rise in the CD4 cell count and fall in HIV viral load. An experienced ophthalmologist performed dilated funduscopy at the time of recruitment and thereafter at 2 weekly intervals and retinal photography was performed at monthly intervals in patients with CMVR. The activity and progression of CMV retinitis was assessed on the basis of the characteristic clinical and photographic findings.
\end{abstract}

Results-34 patients achieved at least 50 CD 4 cells $\times 10^{6} / 1$ at 3 months after initiation of therapy. New diagnoses of CMVR were seen only in the non-responder group $(p=0.085)$. Overall, the relative risk of a new retinitis event in this group was 3.52 $(95 \%$ CI $1.16,10.68)$ at 3 months compared with those patients who were responsive to HAART. 12 of the 63 patients had previous CMVR. Disease progression was associated with non-response to therapy $(p=0.182$ exact). In patients with CMVR the median time to first progression was 18 days $(95 \% \mathrm{CI} 8,91)$ in non-responders and 121 days $(95 \%$ CI $0.59,3.65)$ in responders. By the end of the 2 year follow up period all surviving patients had $>50$ CD4 cells $\times 10^{6} / 1$. No CMV events were seen after 8 months of therapy in either group of patients.

Conclusions-These findings suggest that significant clinical immunorestoration to CMV occurs in response to HAART in patients with CMVR after a lag time of 3-8 months. Initially, a rise in CD4 count is predictive of CMVR response but after the lag period all survivors appear to have developed a clinical immunorestoration to CMV. If HAART is commenced in at risk patients before the development of CMVR the incidence of new disease falls significantly.

(Br f Ophthalmol 1999;83:652-655)

Cytomegalovirus retinitis (CMVR) remains the commonest opportunistic infection in patients with AIDS, occurring with increasing frequency as the CD4 count falls below 100 cells $\times 10^{6} / 1 .{ }^{12}$ Before the use of highly active antiretroviral therapy (HAART), consisting of antinucleoside analogues and proteinase inhibitors, the lifetime risk of developing CMVR was reported to be $44.9 \% .^{3}$ Although in recent years the number of therapeutic options for the treatment of CMVR has increased, treatment remains suboptimal. Despite a good response in the first instance to anti-CMV therapy, CMVR tends to progress. Before the use of HAART the median time to first progression of CMVR with no anti-CMV maintenance therapy was less than 21 days $^{4}$ and with oral ganciclovir maintenance therapy is reported as 57 days. ${ }^{5}$ Most patients with CMVR have good vision at presentation. In a series of 287 patients the median time to vision of $6 / 60$ or worse in an eye with retinitis was 13.4 months. ${ }^{6}$

The HIV proteinase inhibitor indinavir, in combination with nucleoside analogues, has been shown to produce a fall in serum HIV viral load and a sustained rise in CD4+ $\mathrm{T}$ cell count. $^{7}$ The effect of this partial immunorestoration on the incidence of opportunistic diseases in patients with AIDS is still being evaluated but mortality decreases significantly in patients with CMVR treated with HAART. ${ }^{8}$ Early reports by Whitcup et al describe the beneficial effect of HAART on CMVR. ${ }^{9}$ Jacobson and colleagues report on several patients who continued to develop progression of CMVR in the first 2 months of HAART despite rises in CD4 cell count. However, overall these patients also had delayed progression of their CMVR. ${ }^{10}$

The aim of this 2 year prospective study was to assess the effect of starting indinavir (as part of antiretroviral combination therapy) on the incidence and progression of CMVR in a larger cohort of late stage HIV patients.

Patients and methods

Patients with CD4 cell counts below 50 cells $\times 10^{6} / 1$ were recruited to the study between November 1995 and November 1996. All 
Table 1 Summary of new CMV retinitis and progression of CMV retinitis measuring response to therapy as a rise of 50 cells $\times 10^{6} / l$ over 3 months

\begin{tabular}{llcc}
\hline & All patients & Responders & Non-responders \\
\hline No of patients & 63 & 34 & 29 \\
No with previous CMVR & 12 & 6 & 6 \\
No with progression of CMVR & $9(75 \%)$ & $3(50 \%)$ & $6(100 \%)$ \\
No with no previous CMVR & 51 & 28 & 23 \\
No with new CMVR & $3(6 \%)$ & 0 & $3(11 \%)$ \\
No with progression or new CMVR & $12(19 \%)$ & $3(9 \%)$ & $9(31 \%)$ \\
\hline
\end{tabular}

patients were CMV antibody positive and were proteinase naive. Combination antiretroviral therapy was commenced with $800 \mathrm{mg}$ indinavir three times a day, together with a change of nucleoside analogues where possible. The patients were followed prospectively and CD4 cell counts were monitored monthly. Dilated funduscopy was performed at the time of entry into the trial and at 2 weekly intervals thereafter in patients with CMVR. Fundal photography was performed monthly in these patients. All ophthalmological examinations were performed by an experienced ophthalmologist. CMVR was diagnosed on the basis of the typical clinical findings and reactivation and progression monitored in the standard way. ${ }^{11}$ Anti-CMV therapy available during the course of the study included intravenous ganciclovir, foscarnet, and cidofovir, intravitreal ganciclovir, foscarnet, ISIS 2922, and oral ganciclovir, and was assumed to have remained optimal throughout the study.

Examining the change in the patients' CD4 count was used to assess the response to indinavir therapy. Subjects with an increase to 50 cells $\times 10^{6} / 1$ or more, following 3 months of HAART, were classified as "responders". HIV viral load measurements showed significant decreases in the great majority of subjects and was therefore not used to discriminate between patients. Of the 63 patients in this study, there were 34 responders (median increase of 108 cells $\times 10^{6} / 1$, quartiles 72,144 ), and 29 nonresponders (median increase of 20 cells $\times 10^{6} / 1$, quartiles 1 and 33). There was no difference in duration of follow up between the responders and non-responders $(\mathrm{p}=0.92$, Wilcoxon rank sum test).

When frequencies were small, comparison was made by the Fisher's exact two tail test. Otherwise the Cochran-Mantel-Haenszel $\chi^{2}$ test $(\mathrm{CMH})$ was used to construct a test based confidence interval on relative risk estimates. The log rank test was used to assess the time to progression of disease in those patients with CMV retinitis.

\section{Results}

Sixty three patients were recruited into the study, which included 12 patients with previous CMV retinitis. At 3 months, 29 of the patients had achieved greater than 50 CD4 cells $\times 10^{6} / 1$ (responders). Overall, $12(19 \%)$ patients had a new CMV retinitis event or a progression: three $(9 \%)$ of the responders, and nine $(31 \%)$ of the non-responders $(p=0.026$, $\mathrm{CMH}$ : relative risk $3.52,95 \%$ CI $1.16,10.68)$.

In the group with previous CMV retinitis, all of the six non-responders had a disease progression whereas only three of the six

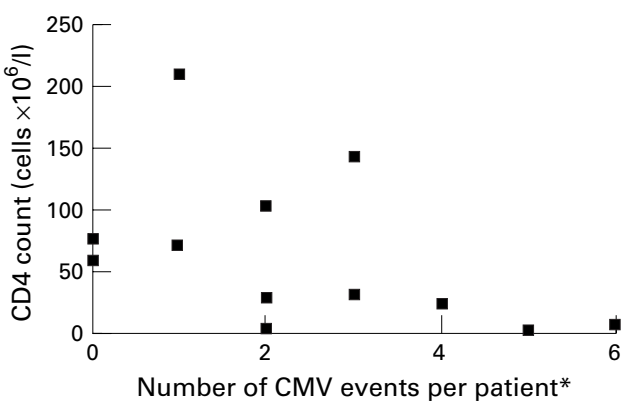

Figure 1 Number of CMV events against CD4 count at 3 months. ${ }^{*}$ There were no CMV events after 8 months.

responders had further retinitis $(\mathrm{p}=0.182$, exact). Three patients $(6 \%)$ with no previous retinitis developed new CMV disease: all were non-responders $(\mathrm{p}=0.085$, exact).

In patients with CMVR, the median time to first progression of CMVR after the start of therapy was 18 days for the non-responders $(95 \%$ CI 8,91$)$ and 121 days for the responders $(95 \%$ CI 51,181$)(p=0.03, \log$ rank test).

In the follow up period after the initial 3 months, none of the responders developed further progressions or new CMV retinitis. In the non-responder group, however, disease progression continued until 8 months, after which time no further CMV episode occurred throughout the 2 year follow up (Table 1). Indeed, in all the surviving patients, the CD4 count had risen to at least 50 cells $\times 10^{6} / 1$ by the end of the study. Analysis of the HIV viral load in this group of patients at 2 years showed no correlation of viraemia with $\mathrm{CD} 4$ count or CMV events.

The number of CMV progressions per year was used to define the event density. A reduction in the CMV retinitis event density was seen in those patients who responded to HAART $(p=0.0036)$. In Figure 1 the total number of CMV events per patient is plotted against their CD4 count at 3 months.

There were six deaths in the original cohort of 63 patients. All were non-responders and died within 6 months of commencing HAART ( $p<0.001$ exact).

In this series, response to HAART was not found to be related to the fall in the CD 4 count preceding therapy, duration of low CD4 count, previous antiretroviral therapy, or to the presence of CMVR before the commencement of therapy.

\section{Discussion}

CMVR progression is stopped by HAART within 100 days in subjects whose CD4 counts rise above 50 cells $\times 10^{6} / 1$ in the first 3 months of therapy. We show that even in the group of patients who initially do not respond to HAART with a rise in the CD4 cell count no further CMV events are seen after 8 months. This indicates that a significant clinical immunorestoration to CMV also occurs in these patients.

The observed decrease in CMV events in these patients is probably associated with a heightened CMV specific $\mathrm{T}$ cells response occurring after initiation of HAART. This 
could result from an increase in $\mathrm{T}$ cell activity, an expansion of a pre-existing (but low frequency) CMV specific $\mathrm{T}$ cell pool, or by the generation of new $\mathrm{T}$ cell clones from the thymus.

Recent studies have shown that it is possible to quantitate specific CMV responses by antigen specific CD4+ lymphocyte responses (upregulation of lymphocyte activation marker CD69+ and production of effector cytokine TNF $\alpha$ ) by flow cytometry. ${ }^{12}{ }^{13}$ Komanduri and co-authors have shown that the loss of CMV specific CD4+ lymphocyte responses can be restored after ganciclovir therapy and HAART and that there is strong correlation between the presence of active end organ disease and reduced specific CD4+ lymphocyte frequencies. ${ }^{14}$ It is important to note that progression of CMVR, post HAART, can occur with higher than expected CD4 cell counts suggesting that the absolute CD4 T cell number increases before $\mathrm{T}$ cell function, or $\mathrm{T}$ cell repertoire, has been restored.

An increased thymic activity has been suggested to occur during HAART which could cause the observed rise in the "naive" (CD45RA) $\mathrm{T}$ cells. ${ }^{15}$ Such a rise in thymically derived $\mathrm{T}$ cell clones might provide the best hope of true immune reconstitution. The repertoire of $\mathrm{T}$ cell specificities, which is decreased during HIV disease, could be broadened providing the immune system with the capability to recognise more potential pathogens. The CD45RA increase is seen at 4-6 months, however, implying that the heightened CMV specific activity, suggested in this study, probably occurs too early to be explained by renewed thymic activity. It may, however, contribute to the later reduction in CMV events seen in the survivors of the non-responder group. Indeed it may be more accurate to call this group late responders to therapy rather than non-responders. In this group no correlation was found between CMVR, HIV viral load, and CD4 count. Other authors have noted this discordance between these factors and it is clear that more clinically applicable measures of CMV specific immunorestoration are still required. ${ }^{16}$

In this study an absolute rise of $\mathrm{CD} 4$ cell count to $>50$ cells $\times 10^{6} / 1$ in the first 3 months of HAART, which was sustained for a further 3 months, was a useful indication of those patients who would develop a significant clinical immunorestoration to CMV and hence may well be a useful predictive marker of those patients who could safely stop anti-CMV therapy. Successful discontinuation of antiCMV therapy using the CD4 cell count as a marker of immunorestoration has already been reported. ${ }^{17-19}$ This study also shows that even in surviving patients who are non-responders sufficient immunuological reconstitution has occurred by 8 months to prevent further CMV events. Hence, with time, they too may be considered candidates for the discontinuation of therapy. The survival issues surrounding discontinuation of anti-CMV therapy in this group of patients are still unclear but the potential improvement in quality of life and reduction in drug associated side effects ensure that studies looking at the discontinuation of anti-CMV therapy will continue.

In the 28 patients without previous CMVR who responded to therapy none developed new disease. In clinical studies the probability of developing CMVR has been shown to be $24.6 \%$ by 4 years after the first CD 4 count $<0.1 \times 10^{9} / 1 .^{20}$ The expected incidence of new CMVR was not seen in patients receiving HAART in this study.

In the pre-HAART era molecular techniques based on quantitative CMV polymerase chain reaction (PCR) made it possible to associate CMV replication with clinical disease. Pertel et al showed that in patients with CD4 counts $<50$ cells $\times 10^{6} / 1$ the risk of developing CMV end organ disease was associated with an increased CMV viral load. ${ }^{21}$ In PCR positive patients, each $0.25 \log _{10}$ increase in viral load increased the risk of $\mathrm{CMV}$ disease (relative hazard 1.37). The value of this technique together with those measuring CMV specific lymphocyte responses needs further analysis if pre-emptive therapy is to be appropriately targeted to at risk patients in the HAART era.

In summary, this study demonstrates the immunological benefit of HAART on the natural history and pathogenesis of CMVR. It not only demonstrates the value of CD4 cell count in predicting a subgroup of patients where new CMVR or CMV progression will cease to occur, but also highlights a second subgroup where CMV events cease at a later date. It may well be that CD4 count alone is a useful predictive marker for the safe discontinuation of anti-CMV therapy in the first group, but highlights the fact that in the second subgroup further evaluation of sensitive microbiological and immunological assays are required. In all patients the long term benefit of HAART and the safety of withdrawal of anti-CMV therapy remains unknown.

We thank A W Sawyer for statistical advice.

1 Gallant JE, Moore RD, Richman DD, et al. Incidence and natural history of cytomegalovirus disease in patients with advanced human immunodeficiency virus disease treated with zidovudine. The Zidovudine Epidemiology Study Group. F Infect Dis 1992;166:1223-7.

2 Kuppermann BD, Petty JG, Richman DD, et al. Correlation between CD4+ counts and prevalence of cytomegalovirus retinitis and human immunodeficiency virus-related noninfectious retinal vasculopathy in patients with acquired immunodeficiency syndrome. Am f Ophthalmol 1993;115: 575-82.

3 Hoover DR, Saah AJ, Bacellar H, et al. Clinical manifestations of AIDS in the era of pneumocystis prophylaxis. tions of AIDS in the era of pneumocystis prophylaxis. 1922-6.

4 Studies of Ocular Complications of AIDS Research Group. Mortality in patients with the acquired immunodeficiency syndrome treated with either foscarnet or ganciclovir for cytomegalovirus retinitis. N Engl F Med 1992;326:213-20.

5 Drew WL, Ives D, Lalezari JP, et al. Oral ganciclovir as maintenance treatment for cytomegalovirus retinitis in patients with AIDS. N Engl F Med 1995;333:615-20.

6 Jabs DA. Ocular manifestations of HIV infection. Trans Am Ophthalmol Soc 1995;93:623-83.

7 Hammer SM, Squires KE, Hughes MD, et al. A controlled trial of two nucleoside analogues plus indinavir in persons with human immunodefeiciency virus infection and CD4 cell counts of 200 per cubic millimeter or less. N Engl f Med 1997;337:725-33.

8 Walsh J, Jones C, Barnes E, et al. Increasing survival in AIDS patients with cytomegalovirus retinitis treated with combipatients with cytomegalovirus retinitis treated with combi-
nation antiretroviral therapy including HIV protease inhibitors. AIDS 1998:12:613 18.

9 Whitcup SM, Fortin E, Nussenblatt RB, et al. Therapeutic effect of combination antiretroviral therapy on cytomegalovirus retinitis [letter]. $\mathscr{F} A M A$ 1997;277:1519-20. 
10 Jacobson MA, Zegans M, Pavan PR, et al. Cytomegalovirus retinitis after initiation of highly active antiretroviral retinitis after initiation of high

11 Holland GN, Buhles WC Jr, Mastre B, et al. A controlled retrospective study of ganciclovir treatment for cytomegalovirus retinopathy. Use of a standardized system for the assessment of disease outcome. Arch Ophthalmol 1989;107 1759-66.

12 Waldrop SL, Pitcher CJ, Peterson DM, et al. Determination of antigen-specific memory effector CD4+ T cell frequencies by flow cytometry: evidence for a novel, antigen specific homeostatic mechanism in HIV-associated immunodeficiency. F Clin Invest 1997;99:1739-50.

13 Prussin C, Metcalfe DD. Detection of intracytoplasmic cytokine using flow cytometry and directly conjugated anti-cytokine antibodies. 7 Immunol Methods $1995 ; 188$ : anti-cyto $117-28$.

14 Komanduri KV, Viswanathan MH, Wieder ED, et al. Restoration of cytomegalovirus-specific CD4+ T-lymphocyte ration of cytomegalovirus-specific $\mathrm{CD} 4+$ 1-lymphocyte
responses after ganciclovir and highly active antiretroviral therapy in individuals infected with HIV-1. Nat Med 1998; therapy in

15 Kelleher A, Carr A, Zaunders J, et al. Alterations in the immune response of human immunodeficiency virus (HIV)-infected subjects treated with an HIV-specific protease inhibitor, ritonavir. F Infect Dis 1996;173:321-9.
16 Kaufmann D, Pantaeleo G, Sudre P, et al. CD-4 cell count in HIV-1 infected individuals remaining viraemic with highly active antiretroviral therapy (HAART). Lancet 1998;351: 723-4.

17 Tural C, Romeu G, Sirera D, et al. Long-lasting remission of cytomegalovirus retinitis without maintenance therapy in human immunodeficiency virus-infected patients. F Infect Dis 1998;177:1080-3.

18 Macdonald JC, Torriani FJ, Morse LS, et al. Lack of reactivation of cytomegalovirus (CMV) retinitis after stopping CMV maintenance therapy in AIDS patients with sustained elevations in CD4 T cells in response to highly active tained elevations in CD $4 \mathrm{~T}$ cells in response to highly
antiretroviral therapy. F Infect Dis 1998;177:1182-7.

19 Vrabec TR, Badassano VF, Whitcup SM. Discontinuation of maintenance therapy in patients with quiescent cytomegalovirus retinitis and elevated CD4+ counts. Ophthalmology 1998;105:1259-64.

20 Bowen E, Sabin C, Wilson P, et al. Cytomegalovirus (CMV) viraemia detected by polymerase chain reaction identifies a group of HIV-positive patients at high risk of CMV disease. Aroup of HIV-positive p

21 Pertel P, Hirschtick RE, Phair J, et al. Risk of developing cytomegalovirus retinitis in persons infected with the human immunodefieciency virus. I Acquir Immune Defic Syndr 1992;5:1069-74 